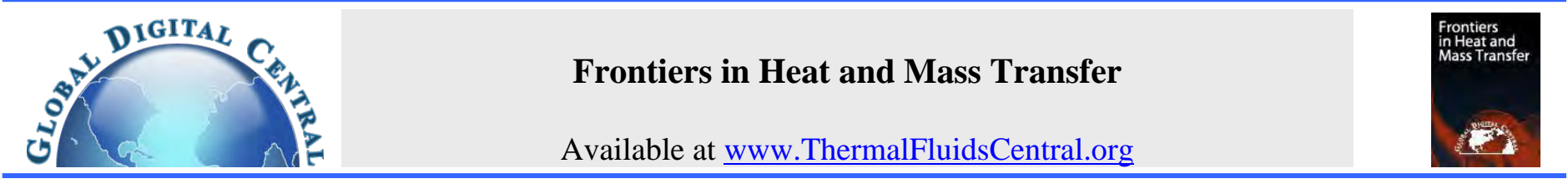

\title{
ENHANCEMENT OF THERMOELECTRIC DEVICE PERFORMANCE THROUGH INTEGRATED FLOW CHANNELS
}

\author{
B. V. K. Reddy, Matthew Barry, John Li, Minking K. Chyu* \\ Department of Mechanical Engineering and Materials Science, University of Pittsburgh, Pittsburgh, PA 15261, USA
}

\begin{abstract}
In this study, the thermoelectric performance of an integrated thermoelectric device (iTED) with rectangular, round end slots, and circular flow channel designs applied to waste heat recovery for several hot stream flow rates has been investigated using numerical methods. An iTED is constructed with p- and n-type semiconductor materials bonded to the surfaces of an interconnector with flow channels drilled through it. This interconnector acts as an internal heat exchanger directing waste heat from the hot stream to thermoelectric elements. The quantity of heat extracted from the waste heat source and the subsequent amount of electrical power generated $\mathrm{P}_{0}$ from the iTED is increased significantly for the circular flow channels, followed by round end slots and rectangular flow channels, respectively. At $R e=100$, the round end slots and the circular flow channels showed nearly 2.6 and 2.9 times increment in $\mathrm{P}_{0}$, and 1.5 and 1.65 times in $\eta$ when compared to the rectangular flow channels values. Conversely, when $R e$ is increased from 100 to 500, the iTED with rectangular flow channels showed 2.67- and 1.6-fold improvement in $\mathrm{P}_{0}$ and $\eta$, respectively. However, the circular configurations showed 2.27- and 1.41-fold increases in $\mathrm{P}_{0}$ and $\eta$ values, respectively. Within the $R e$ range studied, the inclusion of flow channels' pumping power in $\eta$ calculations showed negligible effect. For an iTED with circular flow channels, an increase in a cold side convective heat transfer coefficient $h_{c}$ resulted in an enhancement in $P_{0}$ and $\eta$ values. Besides a $\mathrm{h}_{c}$ effect, the heat loss to the ambient via convective and radiation heat transfer exhibited an increase in $P_{0}$ and decrease in $\eta$.
\end{abstract}

Keywords: Circular, numerical model, performance, rectangular, slots, thermoelectrics, waste-heat recovery.

\section{INTRODUCTION}

Over the last two decades, the increase in human population and demand of a higher quality of life has increased the energy requirements enormously. Thus, the carbon foot-print and waste heat released into the atmosphere are proliferated from both energy consuming and producing equipment. Based on the DOE report by Smith and Thornton (2009), it is approximated that two-thirds of the supplied input energy to these systems is rejected as a waste heat to the atmosphere. Scavenging even a small percentage of waste energy $(5-10 \%)$ can reduce negative environmental impacts and make current equipment more economical. Hence, we are compelled to explore environmentally-benign technologies that can replace and/or enhance the performance of current energy conversion systems while mitigating the emission of greenhouse gases. Thermoelectric devices (TEDs) are one of the prominent technologies to recover the waste heat and convert it into useful electricity. TEDs work as electric power generators when the junction of two different electrically and thermally conductive materials are exposed to a temperature differential (Rowe, 2006). TEDs have no moving parts, are noise-free, scalable, reliable, and compact. The broad range of applications of these devices include temperature measurement, remote radio and satellite power generation stations, refrigeration cooling and waste heat recovery from exhaust streams and other low-grade heat sources, pocket electronics, biothermal batteries to power pacemakers, localized cooling in electronic components and automobile seats.

The maximum conversion efficiency of a TED depends on both materials' intrinsic properties (figure of merit ZT, expressed as $\left(\sigma \alpha^{2} T\right) / \kappa$ ), here $\sigma, \alpha, \mathrm{T}$, and $\kappa$ are the electrical conductivity, Seebeck coefficient, absolute temperature, and total thermal conductivity, respectively) and the hot and cold junction temperatures ( $T_{h}$ and $T_{c}$ ) (Rowe, 2006). Therefore, the TED efficiency can be enhanced either through material strategies (via methods of nano-structuring and fabrication (Sootsman et al., 2009; Tritt, 2011; Biswas et al., 2012) and use of new bulk materials (Rowe, 2006; Poudel et al., 2008)) and/or through the system modeling, design, and optimisation (Caillat et al., 1999; Kaibe et al., 2005; Hodes, 2010; Crane et al., 2012). Caillat et al. (1999) achieved a TED efficiency of $15 \%$ using segmented thermoelectric legs. When cascading TEDs are subjected to $T_{h}=550{ }^{\circ} \mathrm{C}$ and $T_{c}=30{ }^{\circ} \mathrm{C}$, Kaibe et al. (2005) reported an efficiency of $12.1 \%$. Hodes (2010) investigated the optimum leg geometries for maximizing either thermoelectric efficiency or power output in the absence and then presence of interface contact resistances. Recently, Crane et al. (2012) built a full-scale cylindrical-shaped thermoelectric generator using segmented and high-power density elements and obtained a maximum power output of $608 \mathrm{~W}$. They also demonstrated the validity of steady-state and transient TED models by constructing them on cylindrical gas/liquid heat exchangers. Further, Yazawa and Shakouri

${ }^{*}$ Leighton Orr Chair Professor and Chairman and Corresponding author, Email: mkchyu@ pitt.edu, Ph: 412-624-9720 Fax: 412-624-4846. 
(2012) studied the co-optimisation of TEDs by considering the TE leg shape, heat sink, load and contact resistances, and heat losses.

It has been demonstrated that the three-dimensional 3D thermoelectric modeling substantially influences the overall performance of TED (Harris et al., 2006; Hu, 2009; Ziolkowski et al., 2010; Chen et al., 2011). Harris et al. (2006) studied the temperature and electrical field distributions in TEDs using a finite volume numerical model by taking into account both temperature-dependent material properties and contact resistances at the interfaces of the thermoelectric material and conducting shoes. $\mathrm{Hu}$ (2009) analysed the characteristics of two compact gasphase heat exchangers placed on the hot and cold sides of the TED to enhance heat transfer and fluid flow while minimizing thermal stresses. Ziolkowski et al. (2010) investigated the performance of TEDs including the parasitic effects such as convection, radiation and conduction heat bypass. Further, accounting all temperature-dependent material properties and non-linear fluid-thermal-electric coupled mechanisms, Chen et al. (2011) developed a 3D numerical model for TEDs in FLUENTUDS environment. Recently, using 3D numerical methods Reddy et al. $(2012,2013 a)$ studied the enhanced heat transfer characteristics and performance of composite and integrated TEDs applied to waste heat recovery systems.

It is observed from the literature that the conventional TED designs, due to their geometrical structure, induce large amounts of thermal stresses Furthermore, a thermal resistance present at the interfaces of the ceramic plates and the heat sinks. Keeping in mind the minimisation of contact resistances and thermal stresses, recently Reddy et al. $(2012,2013 \mathrm{~b})$ proposed an integrated thermoelectric device (iTED) where each leg is made of semiconductor slices bonded onto a highly conducting inter-connector material with flow channels which act as a heat exchanger between the flowing fluid and thermoelectric materials. It was demonstrated that such a design would increase the performance of iTED with given thermoelectric materials and geometric conditions. Furthermore, the iTED's interconnector flow channel configuration type will play a significant role in achieving higher power output and efficiencies. Therefore, in this study, the theoretical performance of an iTED applied to waste heat recovery with various integrated flow channel designs (rectangular, round end slots and circular) and several hot fluid flow rates have been investigated. Further, the influence of cold wall convective heat transfer conditions and the heat loss to the ambient via the convective and radiation heat transfer on iTED's performance are studied.

\section{GEOMETRY, GOVERNING EQUATIONS, AND SOLUTION PROCEDURE}

Thermoelectric legs made of semiconductor slices bonded onto a highly conducting inter-connector material with a flow channel configuration can be treated as integrated Thermoelectric Devices (iTED). The schematic of such an iTED with a rectangular flow channel configuration is shown in Fig. 1a. It comprises of a vertical leg constructed with p- and n-type semiconductor materials and copper as a inter-connector. Leg with square cross-section $\mathrm{W} \times \mathrm{D}$ and height $H$ is considered. Further, the thickness of $\mathrm{n}$ - and p-type semiconductor slices equal in size $d$ is assumed. As shown in Fig. 1a, the inter-connector with rectangular flow channel grooved through it acts as a heat exchanger between n- and p-type semiconductor slices. The two dimensional view (in the direction of flow) of various flow channel configurations such as rectangular, slots with round end and circular channels are also shown in Fig. 1b. The two copper connectors placed one at the top and one at the bottom of the leg constitute two junctions. The connectors also act as terminals when the device is connected to the load circuitry of resistance $R_{L}$.

As shown in Fig. 1a, a hot fluid with constant inlet temperature $T_{i n}$ and uniform velocity $U$ enters the main flow channel of cross-sectional area $D \times(L-2 d)$, flows through inter-connector's flow channels, and leaves at the exit of the fluid domain. The inner walls and left and right side surfaces of inter-connector are solely accountable for the heat trans-

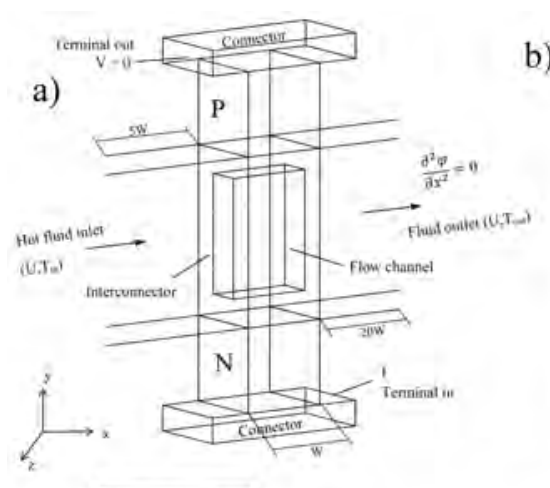

b)
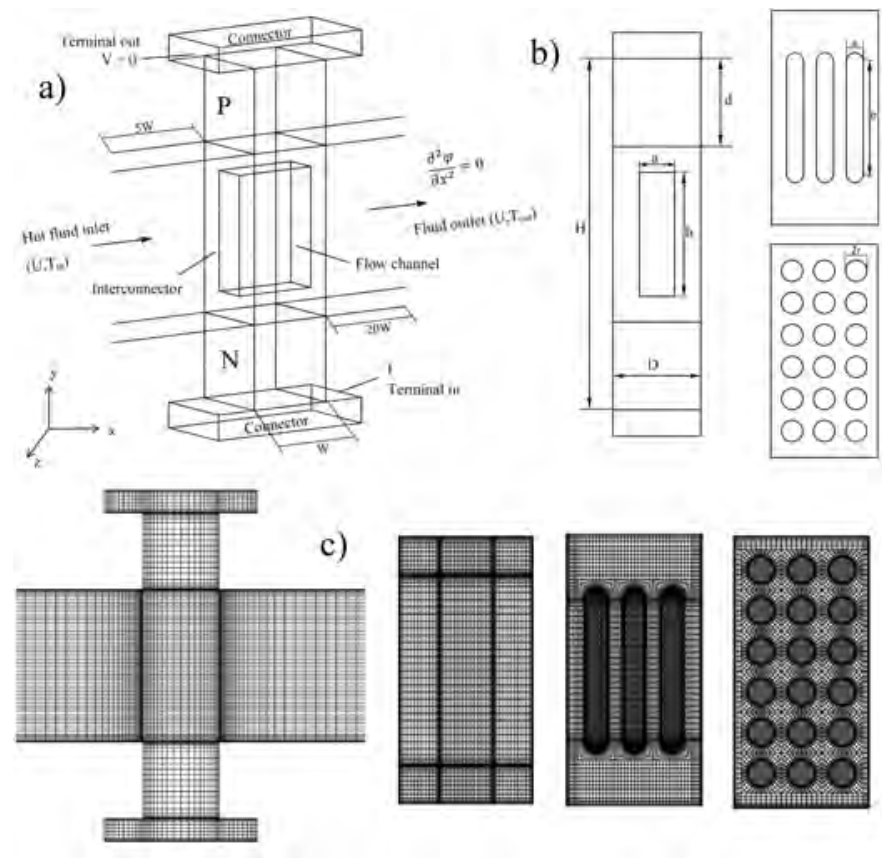

Fig. 1 Schematic of a) an integrated thermoelectric device with b) various flow channel configurations and c) their computational mesh.

fer from the hot fluid to the thermoelectric (TE) elements. The top surface of the upper connector and the lower surface of the bottom connector are exposed to either constant cold temperature $T_{c}$ or convective heat transfer conditions. The remaining surfaces of the iTED are subjected to either adiabatic or convective and radiative heat transfer boundary conditions while keeping the main flow channel walls adiabatic. An upstream buffer length $5 W$ and a downstream buffer zone $20 W$ have been considered to eliminate thermal back diffusion at the inlet and to meet an outflow boundary condition at the outlet of the flow domain, respectively. The iTED connected to a load resistance $R_{L}$ delivers electrical power via the Seebeck voltage produced through a temperature difference $T_{h}-T_{c}$ between the inter-connector channel walls and the cold surface.

The mass, momentum, and heat transport in the fluid regions and the electric current flow and heat transport in the TE materials are governed by the three-dimensional conservation equations subjected to the following assumptions: the thermophysical properties of the fluid are kept constant; the fluid flow is steady, laminar and incompressible; the materials are heterogeneous, isotropic, and the thermoelectric properties are temperature-dependent.

The governing equations for the incompressible fluid flow and heat transport in the fluid region and the thermo-electric coupling effects in the conductor and semiconductor materials under steady-state conditions are written as:

- Continuity equations:

$$
\begin{gathered}
\nabla \cdot \mathbf{v}=0 \text { fluid } \\
\nabla \cdot \mathbf{J}=0 \quad \text { conductor and semiconductor }
\end{gathered}
$$

- Momentum equation:

$$
\rho_{f}(\mathbf{v} \cdot \nabla \mathbf{v})=-\nabla P+\mu \nabla^{2} \mathbf{v}
$$

- Energy equations:

$$
\begin{gathered}
\left(\rho c_{P}\right)_{f}(\mathbf{v} \cdot \nabla T)=\nabla \cdot\left(k_{f} \nabla T\right) \quad \text { fluid } \\
\nabla \cdot(k \nabla T)+\rho \mathbf{J}^{2}=0 \quad \text { conductor }
\end{gathered}
$$




$$
\nabla \cdot(k \nabla T)+\rho \mathbf{J}^{2}-T \mathbf{J} \cdot\left[\left(\frac{\partial \alpha}{\partial T}\right) \nabla T+(\nabla \alpha)_{T}\right]=0 \text { semiconductor (6) }
$$

In the left-hand side of Eq. (6), the second, third, and fourth terms represent the Joule heating, Thomson effect, and Peltier cooling respectively.

The total electric potential calculated using the non-ohmic currentvoltage (Domenicali, 1954) relationship is written as:

$$
\nabla V=\nabla V_{O}+\nabla V_{S}=-\rho \mathbf{J}-\alpha \nabla T
$$

The first and second terms in Eq. (7), denotes the electrostatic and the Seebeck potential distributions, respectively. Here, the electrostatic potential is due to current flowing in the device whereas the Seebeck potential is due to the temperature differential created in the thermoelectric material.

The associated thermal and electrical boundary conditions for Eqs. (1) to (7) with respect to the geometry shown in Fig. 1a are, at terminal 'in': $\mathrm{J}=\frac{V_{0}}{A_{\xi}\left(R_{i}+R_{L}\right)}, \frac{\partial T}{\partial \xi}=0$; at terminal 'out': $V=0, \frac{\partial T}{\partial \xi}=0$; at the main flow channel walls: $\frac{\partial T}{\partial \xi}=0$; at fluid flow inlet: $\mathrm{u}=\mathrm{U}, \mathrm{v}=\mathrm{w}=0, \mathrm{~T}=\mathrm{T}_{i n}$ and at fluid flow outlet: $\frac{\partial^{2} u}{\partial \xi^{2}}=\frac{\partial^{2} T}{\partial \xi^{2}}=v=w=0$. At the interface between the semiconductor and connector or inter-connector materials, the continuity of temperature, current density and the heat flux conditions are imposed (Reddy et al., 2012).

In the above paragraph, $R_{i}$ is the total internal resistance due to nand p-type slices, connectors and inter-connector materials, and $R_{L}$ is the external load resistance. $V_{0}$ is the total built-in open circuit voltage of iTED at no-load condition and it is calculated as the summation of the Seebeck potential at the interfaces of the semiconductor and conductor materials. The Seebeck voltages are evaluated with reference to the hot surface temperature at the interface of the semiconductor and interconnector materials. The total $R_{i}$ and $V_{0}$ of the device are evaluated as:

$$
\begin{array}{r}
R_{i}=\sum_{j=n, p, c, i c} \frac{L_{j}}{A_{j}}\left[\frac{1}{V_{j}} \int_{V_{j}} \rho_{j} d v_{j}\right] \text { and } \\
V_{0}=\sum_{j=n, p} \frac{1}{A s_{j}} \int_{A s_{j}}\left|\alpha_{j}\right| \frac{d T}{d \xi} d A s_{j} .
\end{array}
$$

The boundary conditions at the top surface of the upper connector and the bottom surfaces of the lower connectors:

$$
T=T_{c} \quad \text { or } \quad q^{\prime \prime}=h_{c}\left(T-T_{\infty}\right), \quad \text { and } \quad \frac{\partial V}{\partial \xi}=0,
$$

and at all other iTED surfaces exposed to surroundings:

$$
q^{\prime \prime}=\frac{\partial T}{\partial \xi}=0 \text { or } q^{\prime \prime}=h_{s}\left(T-T_{\infty}\right)+\epsilon \sigma\left(T^{4}-T_{\text {sur }}^{4}\right) \text { and } \frac{\partial V}{\partial \xi}=0 .
$$

Here, $\xi$ denotes the direction normal to the corresponding surface.

The pressure drop $\Delta P / L$, power output $P_{0}$, heat input $Q_{h}$ at the inter-connector walls exposed to hot fluid and the thermoelectric conversion efficiency of iTED are evaluated as:

$$
\begin{gathered}
\frac{\Delta P}{L}=\frac{1}{25 W}\left[\left.\int_{0}^{H-2 d} \int_{0}^{D} P d y d z\right|_{x=0}-\left.\int_{0}^{H-2 d} \int_{0}^{D} P d y d z\right|_{x=25 W}\right] \\
P_{0}=I^{2} R_{L} \\
Q_{h}=-\frac{1}{A s} \int_{A s} k_{f} \frac{\partial T}{\partial \xi} d A s
\end{gathered}
$$

and

$$
\eta=\frac{P_{0}}{Q_{h}}
$$

Using the finite volume formulation of Eqs. (1) to (6) and the constitutive relation (Eq. 7) along with the above mentioned associated boundary conditions, the numerical simulations are performed in the FLUENTUDS (User Defined Scalar) software. The coupling between velocity and
Table 1 Grid independence study for iTED with various flow channel configurations (grid size in bold face is chosen for further simulations) $\left[T_{i n}=550 \mathrm{~K}, T_{c}=300 \mathrm{~K}, \mathrm{~d}=5 \mathrm{~mm}\right.$, and $\left.\mathrm{H}=20 \mathrm{~mm}\right]$.

\begin{tabular}{|c|c|c|c|c|c|c|}
\hline \multicolumn{7}{|c|}{ Rectangular duct $\left[R e=400, R_{L}=4.69 \times 10^{-3} \Omega\right]$} \\
\hline Cells & $P_{0}, \mathrm{~W}$ & $\begin{array}{c}\text { \% error } \\
\text { in } P_{0}\end{array}$ & $Q_{h}, \mathrm{~W}$ & $\begin{array}{c}\text { \% error } \\
\text { in } Q_{h}\end{array}$ & $\eta, \%$ & $\begin{array}{c}\% \text { error } \\
\text { in } \eta\end{array}$ \\
\hline 300124 & 0.0739 & & 1.6977 & & 4.3513 & \\
633600 & 0.0724 & 1.998 & 1.6821 & 0.929 & 4.3057 & 1.060 \\
$\mathbf{1 1 3 9 1 5 2}$ & 0.0714 & 1.445 & 1.6714 & 0.642 & 4.2716 & 0.797 \\
2000922 & 0.0720 & 0.859 & 1.6803 & 0.534 & 4.2857 & 0.327 \\
\hline Multiple slots with rounded end $\left[R e=400, R_{L}=5.12 \times 10^{-3} \Omega\right]$ \\
\hline 598200 & 0.1593 & \multicolumn{1}{|c|}{2.6052} & & 6.1140 & \\
$\mathbf{1 1 7 5 5 0 4}$ & 0.1590 & 0.151 & 2.6027 & 0.097 & 6.1107 & 0.054 \\
2272752 & 0.1595 & 0.288 & 2.6137 & 0.420 & 6.1026 & 0.133 \\
3307802 & 0.1583 & 0.739 & 2.6029 & 0.415 & 6.0830 & 0.323 \\
\hline \multicolumn{7}{|c|}{ Circular ducts $\left[R e=500, R_{L}=5.29 \times 10^{-3} \Omega\right]$} \\
\hline 948960 & 0.2008 & \multicolumn{7}{|c|}{3.0114} & \multicolumn{3}{|c|}{6.6675} & \\
$\mathbf{1 8 2 2 9 7 6}$ & 0.1983 & 1.259 & 2.9919 & 0.653 & 6.6276 & 0.602 \\
3723840 & 0.1978 & 0.230 & 2.9901 & 0.058 & 6.6162 & 0.172 \\
\hline
\end{tabular}

pressure is handled by using the SIMPLE algorithm. The convective and diffusive terms are discretised with a power law scheme and the pressure term is handled with a standard scheme. The geometric models and mesh are generated in Gambit 2.4. The Seebeck and Ohmic potential distributions (Eq. (7)) and continuity of current density (Eq. (2)) are evaluated using UDS fields. The Ohmic heating, Peltier and Thomson effects are modeled as source terms in the energy equation (Eq. (6)). The electric current is calculated based on the open-circuit Seebeck voltage produced at a given load resistance $R_{L}$. Additional details on implementation of numerical model for TED in the FLUENT-UDS environment has been given in the articles (Chen et al., 2011; Reddy et al., 2012). The convergence criteria for mass and momentum, energy, and current density, Seebeck and Ohmic electric potentials are set as $10^{-5}, 10^{-15}$, and $10^{-10}$, respectively. After performing a grid independence study, suitable grid sizes were chosen for various integrated flow channel configurations and these are depicted in Table 1. The orthogonal, nonuniform grid is used, and for brevity, the computational mesh for an iTED along the front and side views is shown in Fig. 1c. Furthermore, the thermoelectric model has been validated with published results and the details are given in our earlier works (Reddy et al., 2012, 2013a).

\section{RESULTS AND DISCUSSION}

The performance of an integrated thermoelectric device (iTED) for various flow channel configurations in terms of power output $P_{0}$, heat input $Q_{h}$, conversion efficiency $\eta$, electric current $I$, Seebeck and Ohmic voltages $V$, and pressure drop $\Delta P / L$ with several hot fluid flow rates ( $50 \leq R e \leq 500$ ) has been investigated using numerical methods. The rectangular, round end slots, and circular flow channels within the interconnector acting as an internal heat exchanger for the hot fluid flow are considered in the present study. Further, the effects of cold surface boundary condition (constant temperature and convective heat transfer conditions) on the performance of iTED with circular flow channel configuration alone has been studied when the remaining surfaces, except the flow channel walls, exposed to either adiabatic or convective and radiation conditions. The iTED made of n- $\left(75 \% \mathrm{Bi}_{2} \mathrm{Te} 25 \% \mathrm{Bi} \mathrm{i}_{2} \mathrm{Se}\right)$ and p-type (25\% $\mathrm{Bi}_{2} \mathrm{Te}_{3} 75 \% \mathrm{Sb}_{2} \mathrm{Te}_{3}(1.75 \%$ excess $\mathrm{Se})$ ) semiconductor materials, copper as connector and interconnector, and the air as hot fluid have been considered. The thermo-electrical properties of the semiconductor and conductor materials are varied with temperature (Reddy et al., 2012), and the thermo-physical properties of air are kept constant. Fur- 


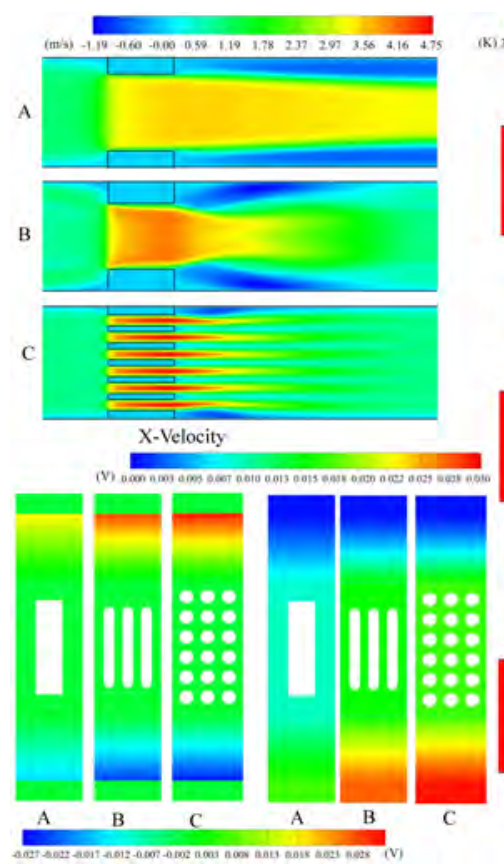

Secbeck voltage Ohmic voltage

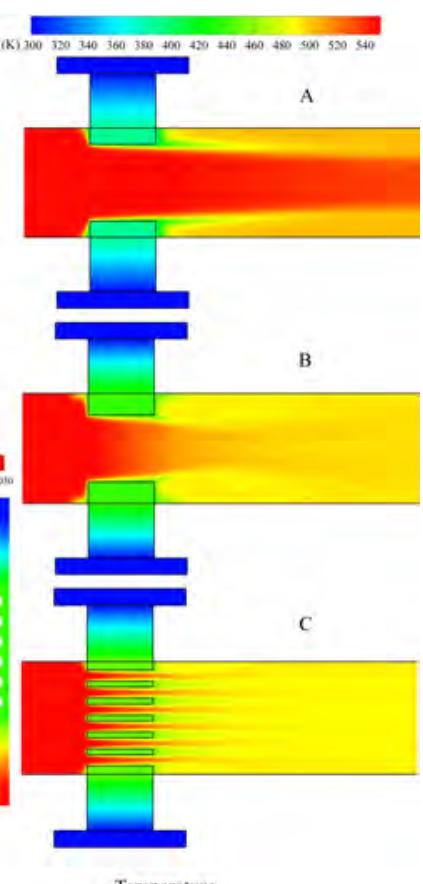

Temperature

Fig. 2 The distributions of $x$-velocity, temperature, Seebeck and Ohmic voltage for various integrated flow channel configurations A) rectangular B) slots with round end and C) circular.

thermore, to achieve maximum $P_{0}$ from an iTED, the load resistance $R_{L}$ equaling to the total internal resistance $R_{i}$ of the device (Eq. (8)) is used in the numerical simulations.

The geometrical dimensions: height $H=20 \mathrm{~mm}$, depth $D=5 \mathrm{~mm}$, connector thickness $t=1.5 \mathrm{~mm}$ and length $L=10 \mathrm{~mm}$, the semiconductor and inter-connector width $W=5 \mathrm{~mm}$, and semiconductor slice thickness $d=5 \mathrm{~mm}$ are maintained constant in the reported results. The hot fluid inlet temperature $T_{i n}=550 \mathrm{~K}$ and cold wall temperature $T_{c}=300 \mathrm{~K}$ are invariant. Further, the ratio of flow cross-sectional areas of an integrated fluid flow channels and the main flow channel is kept fixed and it is expressed as

$$
\phi_{F A}=\frac{A_{c}}{A}
$$

Here, $A$ and $A_{c}$ are the cross-sectional areas of main flow channel $D \times$ $(H-2 \times d)$ and the integrated flow channels, respectively. For various flow channel configurations as shown in Fig. 1b, the $A_{c}$ is evaluated as

$$
A_{c}= \begin{cases}a \times b & \text { Rectangular } \\ n_{s}\left[a_{s} \times b_{s}+\frac{1}{4} \pi a_{s}^{2}\right] & \text { Slots with round end } \\ n_{c} \pi r^{2} & \text { Circular. }\end{cases}
$$

In Eq. (16), $n_{s}, n_{c}$, and $r$ denote the number of slots, number of circles, and the circle radius, respectively. In the present study, $n_{s}=3$ and $n_{c}=$ $6 \times 3$ are used. While maintaining $\phi_{F A}=0.283$ invariant, the dimensions of the various flow channel designs are calculated using Eq. (16).

$\mathrm{X}$-velocity, temperature, and Seebeck and Ohmic voltage distributions for integrated rectangular, round end slots, and circular flow channel configurations with hot fluid flow rate $R e=300$ are shown in Fig. 2, whereas the same variable contours for circular flow channels alone with two $R e$ values are depicted in Fig. 3. The x-velocity and temperature distributions are depicted on z-plane sectioned at $2.5 \mathrm{~mm}$ while the Seebeck and Ohmic voltage contours are shown on x-plane at $27.5 \mathrm{~mm}$. Further, to show velocity and temperature contours in Figs. 2 and 3, an up-stream and down-stream lengths of $W$ and $5 W$, respectively have been taken. At $\phi_{F A}=0.283$ and a given $R e$ value, it is observed that the flow channel

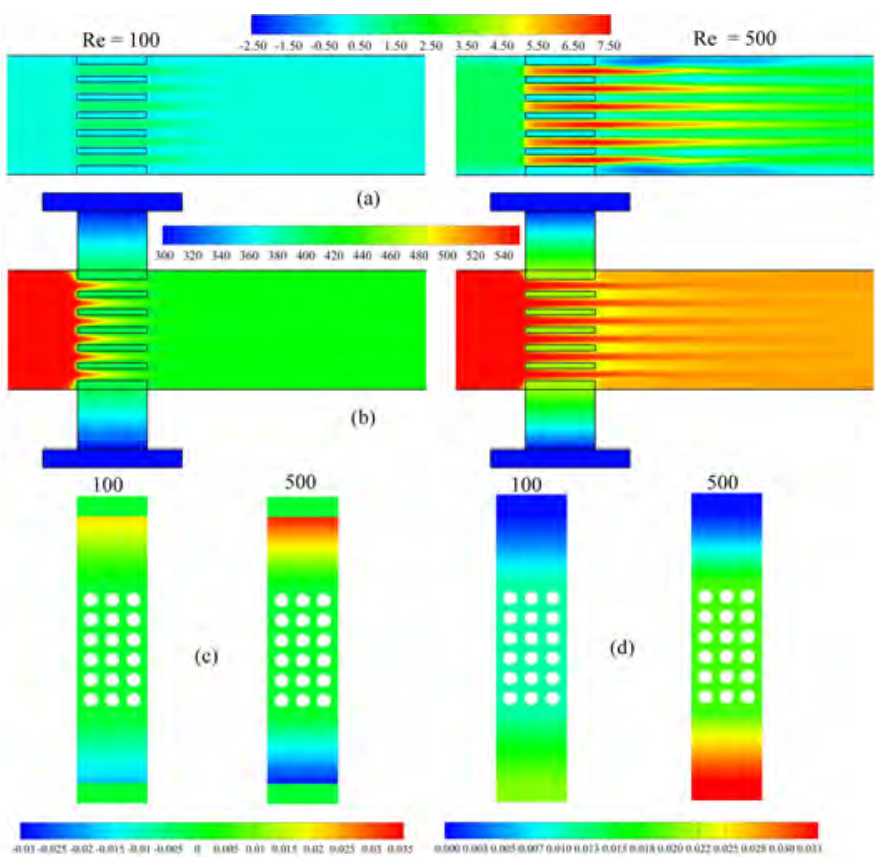

Fig. 3 The contours of a) x-velocity b) temperature c) Seebeck and d) Ohmic voltages for integrated circular flow channels at $R e=100$ and 500 values.

type has a substantial effect on fluid flow, heat transfer, and electric characteristics of iTED. As seen from Fig. 2, in the integrated flow channels, the fluid's average velocity increases and the bulk temperature decreases as the channel configuration varies from rectangular to circular and this subsequently resulted in enhanced heat transfer to the thermoelectric elements. The reason for enhanced heat transfer is due to the increase in the flow channel's surface area with decrease in size of the flow channels. For instance, for the flow configurations studied here, the surface area of round end slots and circular flow channels are 2.16 and 3.12 times higher when compared to the rectangular flow channels, respectively. Furthermore, as shown in Fig. 3 for the circular flow channels, an increment in temperature gradients near the flow channel walls is resulted in an increase in $R e$ from 100 to 500 . This increase in temperature gradients promotes higher convective heat transfer rates and thus in turn results in a reduction of iTED's total thermal resistance value. The recirculation zones and the flow patterns formed in the down stream of integrated flow channels have negligible influence on the thermo-electric performance of iTED, however they exhibit considerable effect on the total pressure drop experienced in their respective channels.

It is seen that an increase in heat transfer area of the flow channels either through round end slots or circular channels as well as the increase in $R e$ resulted in larger temperature gradients in the thermoelectric (TE) elements. As shown in Figs. 2 and 3, this increase in temperature gradients generates higher Seebeck voltages in the TE elements. In each TE element, the Seebeck voltage drop is calculated with respect to the copper material. However, the Ohmic voltage distribution can be obtained through the produced electric current $I$ when the iTED terminals are connected to load circuitry of resistance $R_{L}$. At $R e=300$, the 2.16 and 3.12 times increase in heat transfer area of slots with round end and circular flow channels, respectively, results in 1.6 and 1.74 times increase in Seebeck voltage values when compared to the rectangular flow channel's value. Furthermore, as the flow channel configuration changes from rectangular to circular, the Ohmic voltage drop increased from 0.0168 to $0.0299 \mathrm{~V}$; on the other hand, as Re increased from 100 to 500 for the circular flow channel, the Ohmic voltage drop raised from 0.0206 to 0.0333 . It is also noticed that with $R_{L}=R_{i}$ condition, the Seebeck co- 

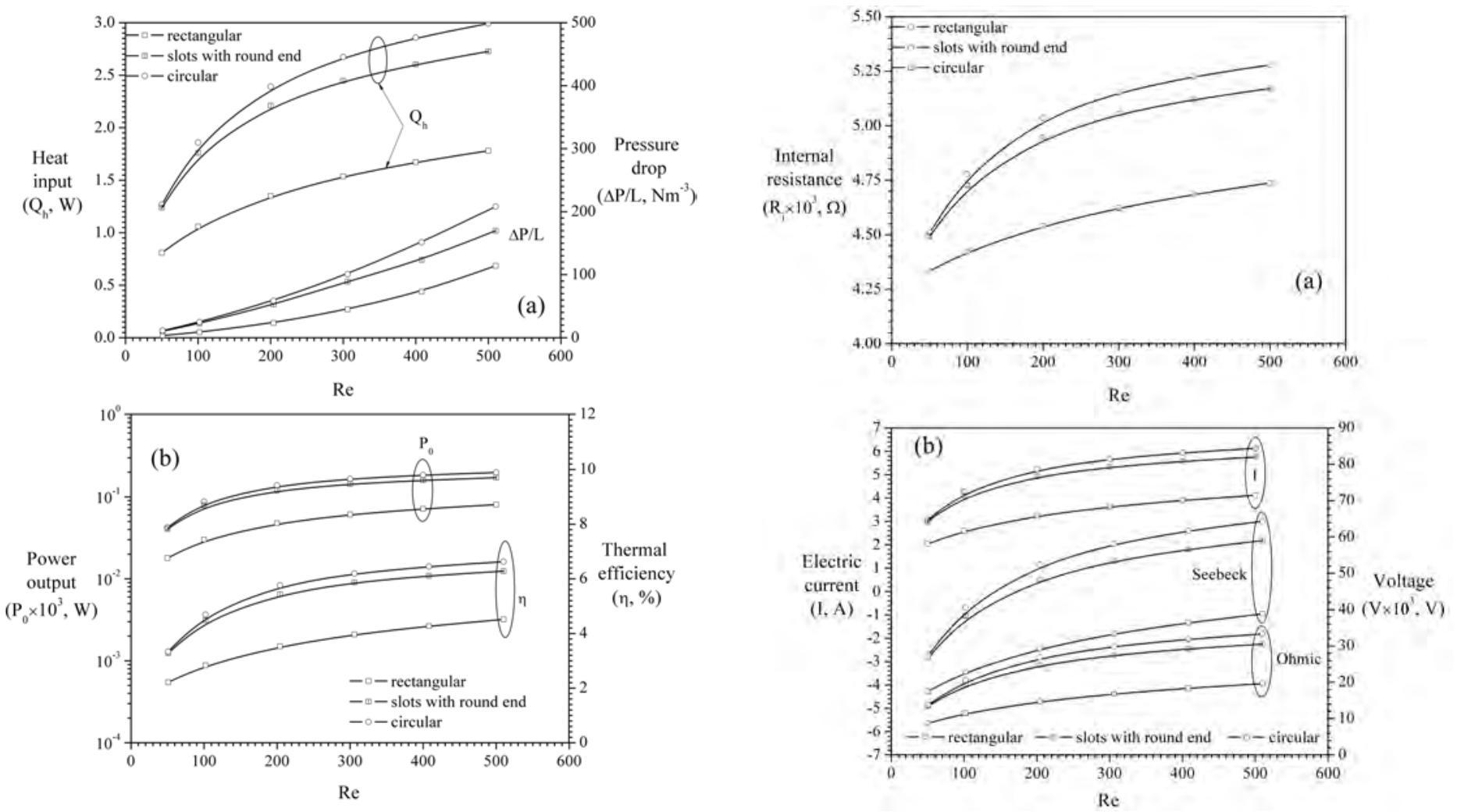

Fig. 4 Effects of $R e$ on a) heat input and pressure drop and b) power output and thermal efficiency for flow channel configurations.

efficients and temperature gradients are only responsible for the Ohmic voltage drop variations and it is independent of $\mathrm{R}_{i}$ values.

The response of total internal resistance $R_{i}$ and produced electric current $I$ and Seebeck and Ohmic voltages $V$ with $R e$ for various integrated flow channel configurations is presented in Figs. $4 \mathrm{a}$ and b, respectively. The rise in temperature gradients in thermoelectric elements with an increase in $R e$ produces larger Seebeck voltages and thus Ohmic voltages (as seen in Fig. 4a). Irrespective of $R e$ value, the circular flow channels showed higher $V-I$ values, followed by round end slots and rectangular flow channels, respectively. It is noticed that $I$ and $V$ exhibited similar trend with $R e$. At a given $R e$, the Ohmic voltage drop is higher than the Seebeck voltage drop value. Furthermore, from Fig. 4b, the $R e$ in the specified range $50 \leq R e \leq 500$ has minimal effect on $R_{i}$ predictions and this is attributed to variation of electrical resistivity behaviour with temperature. However, at a given $R e$ value, a minor increment in $\mathrm{R}_{i}$ is noticed when the iTED's flow channels are varied from rectangular to circular flow configurations.

Figures 5a and b show the results of heat input $Q_{h}$ and pressure drop $\Delta P / L$, and power output $P_{0}$ and thermal efficiency $\eta$, respectively with $R e$ for different integrated flow channel configurations. Irrespective of the flow channel design type, an increase in $R e$ shows an enhancement in $Q_{h}, \Delta P / L, P_{0}$ and $\eta$ predictions. The increase in $R e$ promotes higher heat transfer rate from the hot fluid to TE elements $\mathrm{Q}_{h}$ and this subsequently assists in achieving larger Seebeck voltages (Fig. 4b) and thus results in higher $P_{0}$ and $\eta$ (as seen in Fig. 5b) values. For instance, at $R e=500$ the rectangular flow channel configuration showed 1.68-, 2.6-, and 1.6 times increment in $\mathrm{Q}_{h}, \mathrm{P}_{0}$ and $\eta$, respectively when compared to the values at $R e=100$. Conversely, the round end slots and circular flow channel configurations produced 1.55-, 2.17-, and 1.4 fold, and 1.61-, 2.27-, and 1.41 times increase $\mathrm{Q}_{h}, \mathrm{P}_{0}$ and $\eta$, respectively at $R e=500$ in comparison to values at $R e=100$. For a given integrated

Fig. 5 Effects of Re on a) internal resistance and b) electric current, Seebeck and Ohmic voltages for flow channel configurations.

flow channel configuration, the pressure drop $\Delta P / L$ measured using the Eq. (11) is shown a non-linear behaviour with $R e$ and it is due to the inclusion of additional pressure drop anticipated from the recirculation zones and flow patterns formation in the down-stream and up-stream of integrated flow channels. At given $R e$ value, the circular flow channel configuration displayed higher $\Delta P / L$, and the rectangular flow channel configuration showed the lower $\Delta P / L$ value. It is observed that when $R e$ is less than 200, it has significant effect on device characteristics. It is due to the mutual interplay of temperature gradients and the thermo-electrical property dependency with temperature. It can also noticed that within the $R e$ range, the consideration of pumping power in the $\eta$ calculations has negligible effect.

It is inferred that the iTED with circular flow channel configuration always performs better when compared to other two configurations, i.e. round end slots and rectangular channels. Further, at given $R e$ values, the discrepancy between the performance parameters of circular and round end slots flow channels is less when compared to the discrepancy between the circular and rectangular flow channel configurations values. However, this discrepancy increases minimally with an increase in $R e$. It is also surmised that the number of times increase in integrated heat transfer surface area of channels via round end slots and circular flow channels is not proportional to the increase in the performance parameters.

Figure 6 depicts the effects of cold side convective heat transfer coefficient $h_{c}\left(10 \leq h_{c} \leq 10^{3}\right)$ on $Q_{h}, R_{i}, P_{0}, \eta, I$ and $V$ for an iTED with circular flow channel configuration. In Fig. 6 , the data with filled bullets represents when the iTED walls (except the cold walls and the main flow channel walls) are subjected to adiabatic condition $\left(h_{s}=0\right)$. For given hot fluid flow rate $R e=500$ and cold fluid temperature $T_{\infty}=$ $300 \mathrm{~K}$, A rise in $h_{c}$ shows an improvement in $Q_{h}, P_{0}, \eta, I$ and $V$ values. However, the $R_{i}$ drops as $h_{c}$ increases. It is noticed that as $h_{c}$ increases the cooling heat transfer rate also increases. Therefore, to achieve energy conservation, the heat transfer from hot fluid to iTED also increases 

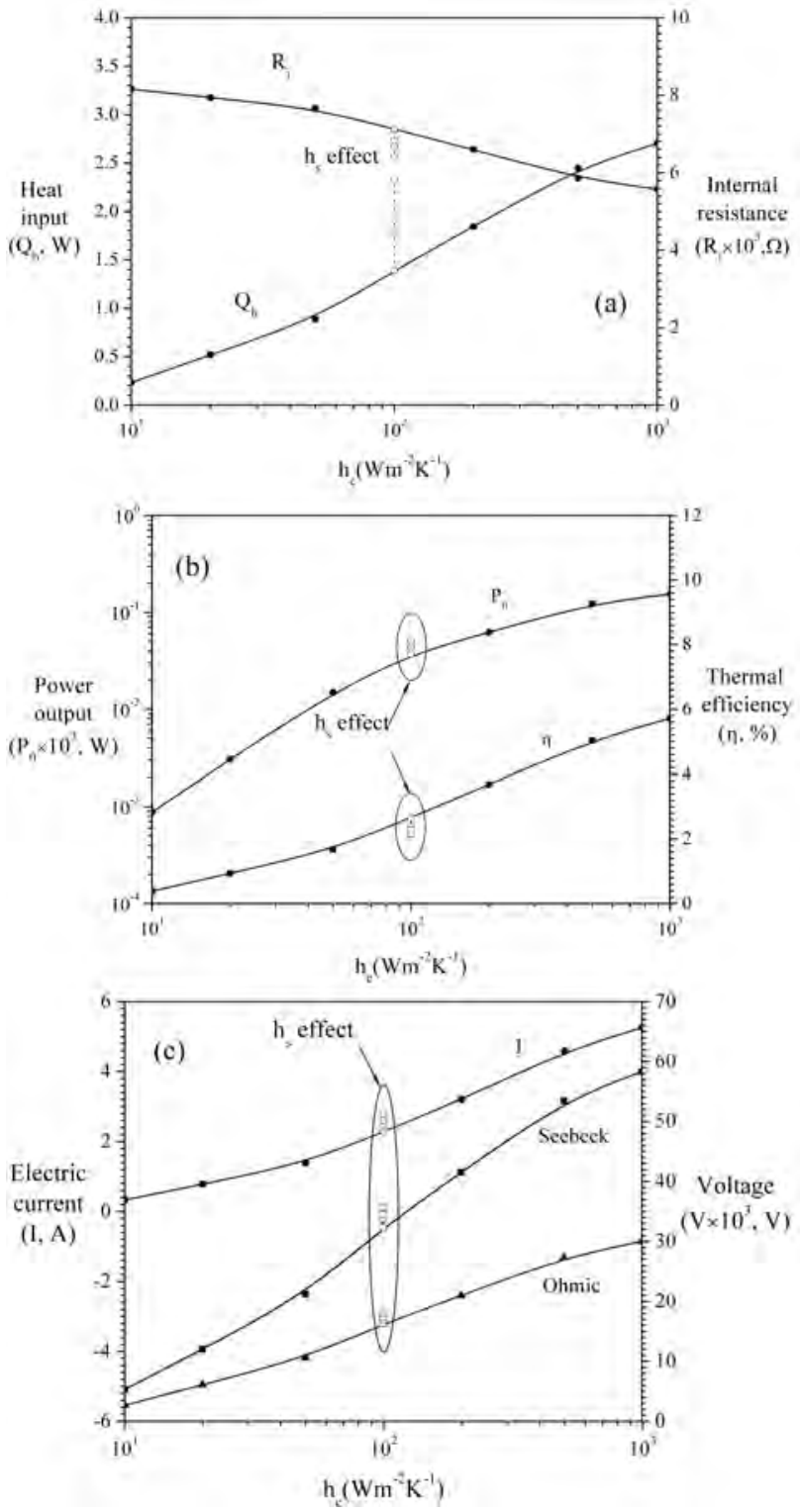

Fig. 6 The influence of cold side convective heat transfer coefficient $h_{c}$ on a) heat input and internal resistance b) power output and thermal efficiency and c) electric current, Seebeck, and Ohmic voltages.

and this results in higher temperature gradients in TE elements. Further, this increase in temperature gradients showed an enhancement in iTED performance parameters. It is also shown in Fig. 6 the influence of convective and radiation heat transfer $h_{s}\left(0,5,10\right.$, and $\left.20 \mathrm{Wm}^{-2} \mathrm{~K}^{-1}\right)$ on iTED performance at $\mathrm{h}_{c}=10 \mathrm{Wm}^{-2} \mathrm{~K}^{-1}$ (the data with empty bullets). Here, the surrounding temperature $T_{\text {sur }}=300 \mathrm{~K}$ is fixed and the emissivities for the semiconductor and the highly polished copper conductor are taken as 0.45 and 0.03 , respectively. It is observed that the rise in $P_{0}$ and $Q_{h}$ and decrease in $\eta$ with an increase in $h_{s}$ values. The heat loss from the walls exposed to ambient resulted in larger $Q_{h}$ predictions; however, the decrease in $R_{i}$ and larger temperature gradients showed enhancement in $P_{0}$ values.

\section{CONCLUSIONS}

The performance of an integrated thermoelectric device (iTED) with various flow channel (rectangular, round end slots, and circular) configurations acting as internal heat exchangers between hot fluid and thermoelec-

tric elements for different flow flow rates $R e$ has been investigated using numerical simulations. Further, the influence of cold wall convective heat transfer conditions and the heat loss to the ambient via the convective and radiation heat transfer on iTED's performance with circular flow channel configuration has been studied.

It is observed that the integrated flow channel configuration type and $R e$ have a substantial effect on iTED's performance. At a given flow channel cross-sectional areas ratio $\phi_{F A}=0.283$, irrespective of $R e$ value, an iTED with circular flow channel configuration showed higher power output $\mathrm{P}_{0}$, efficiency $\eta$, and pressure drop values, followed by round end slots and then rectangular configurations, respectively. This behaviour is attributed to increase in flow channels' surface area with decrease in channel size.

The 2.16 and 3.12 fold increase in heat transfer surface area of the round end slots and the circular flow channels, respectively resulted in 1.6 and 1.74 times increase in Seebeck voltages, 2.36 and 2.72 times in $\mathrm{P}_{0}$, and 1.48 and 1.56 times in $\eta$ values when compared to the rectangular flow channel configuration values at $R e=300$ with inlet temperature $T_{i n}=550 \mathrm{~K}$ and cold surface temperature $T_{c}=300 \mathrm{~K}$ conditions.

When $R e$ increased from 100 to 500, the rectangular flow channel configuration showed 2.67- and 1.6-times improvement in $\mathrm{P}_{0}$ and $\eta$, respectively. However, the round end slots and circular flow channels depicted 2.17- and 1.4 fold, and 2.27- and 1.41-fold increase in $\mathrm{P}_{0}$ and $\eta$ values. For given $R e$ and flow channel configuration, the produced current and Seebeck and Ohmic voltages exhibited similar behaviour as that of $\mathrm{P}_{0}$ and $\eta$. Due to mutual interplay of temperature gradients in thermoelectric material and the temperature dependency of the thermo-physical properties, the discrepancy in the performance of circular and slots with round end flow channels is less when compared to the discrepancy values between the circular and rectangular flow channel configurations.

For an iTED with circular flow channel configuration when the walls except the cold walls and the main flow channel walls are exposed to adiabatic conditions $\left(\mathrm{h}_{s}=0\right)$, an increase in $h_{c}$ resulted in enhancement in heat output $Q_{h}, P_{0}$, and $\eta$ values. In addition to $\mathrm{h}_{c}$ effect, the rise in $h_{s}$ (heat loss to ambient) showed an increase in $P_{0}$ and $Q_{h}$ and decrease in $\eta$ values.

It has shown that under given set of operating conditions, the iTED with circular flow configuration performed better when compared to round end slots and rectangular flow channel configurations. Furthermore, within in the $R e$ range studied, the consideration of flow channel pumping power in $\eta$ evaluations has showed negligible effect.

\section{NOMENCLATURE}

$A$

$A_{s}$

$c_{P}$

$d$

$D$

$D_{h}$

$h$

$H$

I

$J$

J

$k$

$L$

$N$

$P$

$P_{0}$

$q^{\prime \prime}$

$Q$

$R$

$R e$ cross-sectional area $\left(\mathrm{mm}^{2}\right)$

surface area $\left(\mathrm{mm}^{2}\right)$

specific heat of fluid $(\mathrm{J} / \mathrm{kg} \cdot \mathrm{K})$

size of semiconductor material $(\mathrm{mm})$

depth of the thermoelectric leg $(\mathrm{mm})$

hydraulic diameter of main flow channel $\left(\frac{4(L-2 d) \times D}{2(L-2 d+D)}\right)(\mathrm{mm})$

convective heat transfer coefficient $\left(\mathrm{W} / \mathrm{m}^{2} \cdot \mathrm{K}\right)$

height of the leg ( $\mathrm{mm})$

electric current $(\mathrm{A})$

electric current density $\left(\mathrm{A} / \mathrm{m}^{2}\right)$

current density vector

thermal conductivity $(\mathrm{W} / \mathrm{m} \cdot \mathrm{K})$

length (mm)

number of thermoelectric modules

pressure $\left(\mathrm{N} / \mathrm{m}^{2}\right)$

power output $I^{2} R_{L}(\mathrm{~W})$

heat flux $\left(\mathrm{W} / \mathrm{m}^{2}\right)$

heat transfer $(\mathrm{W})$

electric resistance $(\Omega)$

Reynolds number $\rho U D_{h} / \mu$ 


$\begin{array}{ll}T & \text { temperature }(\mathrm{K}) \\ U & \text { inlet velocity }(\mathrm{m} / \mathrm{s}) \\ \mathbf{v} & \text { velocity vector } \\ V & \text { voltage }(\mathrm{V}) \\ u, v, w & \text { velocities in } \mathrm{x}, \mathrm{y}, \mathrm{z} \text { directions }(\mathrm{m} / \mathrm{s}) \\ W & \text { width }(\mathrm{mm}) \\ x, y, z & \text { coordinates }(\mathrm{mm}) \\ \text { Greek } & \text { Symbols } \\ \alpha & \text { Seebeck coefficient }(\mathrm{V} / \mathrm{K}) \\ \mu & \text { dynamic viscosity }\left(\mathrm{N} \cdot \mathrm{s} / \mathrm{m}^{2}\right) \\ \rho & \text { electrical resistivity }(\Omega \cdot \mathrm{m}) \\ \rho_{f} & \text { density of fluid }\left(\mathrm{kg} / \mathrm{m}^{3}\right) \\ \eta & \text { conversion efficiency } \\ \text { Subscripts } & \\ \text { c } & \text { cold wall/conductor } \\ \text { in } & \text { inlet } \\ \mathrm{f} & \text { fluid } \\ \text { h } & \text { hot wall } \\ \text { i } & \text { internal/integrated } \\ \text { ic } & \text { inter-connector } \\ \mathrm{L} & \text { load } \\ \mathrm{n} & \text { n-type semiconductor } \\ \mathrm{O} & \text { Ohmic potential } \\ \mathrm{p} & \text { p-type semiconductor } \\ \mathrm{S} & \text { Seebeck potential } \\ \text { surr } & \text { surroundings } \\ \infty & \text { ambient } \\ & \end{array}$

\section{REFERENCES}

Biswas, K., He, J., Blum, I.D., Wu, C., Hogan, T.P., Seidman, D.N., Dravid, V.P., and Kanatzidis, M.G., 2012, "High-Performance Bulk Thermoelectrics with All-Scale Hierarchical Architectures," Nature, 489, 414-418. http://dx.doi.org/10.1038/nature11439.

Caillat, T., Fleurial, J.P., Snyder, G.J., Zoltan, A., Zoltan, D., and Borshchevsky, A., 1999, "Development of a High Efficiency Thermoelectric Unicouple for Power Generation Applications," Proceedings of the XVllI International Conference on Thermoelectrics, Baltimore, USA. http://trs-new.jpl.nasa.gov/dspace/bitstream/2014/18364/1/99-1841.pdf.

Chen, M., Rosendahl, L.A., and Condra, T., 2011, "A Three-Dimensional Numerical Model of Thermoelectric Generators in Fluid Power Systems," International Journal of Heat and Mass Transfer, 54, 345-355. http://dx.doi.org/10.1016/j.ijheatmasstransfer.2010.08.024.

Crane, D.T., Koripella, C.R., and Jovovic, V., 2012, "Validating SteadyState and Transient Modeling Tools for High-Power-Density Thermoelectric Generators," Journal of E, 41(6), 1524-1534. http://dx.doi.org/10.1007/s11664-012-1955-3.

Domenicali, C.A., 1954, "Stationary Temperature Distribution in an Electrically Heated Conductor," Journal of Applied Physics, 25, 1310-1311. http://dx.doi.org/10.1063/1.1721551.

Harris, R., Hogan, T., Schock, H.J., and Shih, T.I.P., 2006, "Heat Transfer and Electric Current Flow in a Thermoelectric Couple," 44th Aerospace Sciences Meeting and Exhibition, 575, AIAA, Reno, Nevada. http://dx.doi.org/10.2514/6.2006-575.
Hodes, M., 2010, "Optimal Pellet Geometries for Thermoelectric Power Generation," IEEE Trans Components and Packaging Technologies, 33, 307-318.

http://dx.doi.org/10.1109/TCAPT.2009.2039934

Hu, K.S.Y., 2009, Heat Transfer Enhancement in Thermoelectric Power Generation, M.s. thesis, Iowa State University.

http://lib.dr.iastate.edu/etd/12196.

Kaibe, H., Aoyama, I., Mukoujima, M., Kanda, T., Fujimoto, S., Kurosawa, T., Ishimabushi, H., Ishida, K., Rauscher, L., Hata, Y., and Seijirou, 2005, "Development of Thermoelectric Generating Stacked Modules Aiming for $15 \%$ of Conversion Efficiency," Int. Conference on Thermoelectrics, 227-232, IEEE. http://dx.doi.org/10.1109/ICT.2005.1519929.

Poudel, B., Hao, Q., Ma, Y., Lan, Y., Minnich, A., Yu, B., Yan, X., Wang, D., Muto, A., Vashaee, D., Chen, X., Liu, J., Dresselhaus, M.S., Chen, G., and Ren, Z., 2008, "High-Thermoelectric Performance of Nanostructured Bismuth Antimony Telluride Bulk Alloys," Science, 320(5876), 634-638. http://dx.doi.org/10.1126/science.1156446.

Reddy, B.V.K., Barry, M., Li, J., and Chyu, M.K., 2012, "ThreeDimensional Multiphysics Coupled Field Analysis of an Integrated Thermoelectric Device," Numerical Heat Transfer Part A, 62, 933-947. http://dx.doi.org/10.1080/10407782.2012.715988.

Reddy, B.V.K., Barry, M., Li, J., and Chyu, M.K., 2013a, "Mathematical Modeling and Numerical Characterisation of Composite Thermoelectric Devices," International Journal of Thermal Sciences, 67, 53-63. http://dx.doi.org/10.1016/j.ijthermalsci.2012.11.004.

Reddy, B.V.K., Barry, M., Li, J., and Chyu, M.K., 2013b, "Thermoelectric Performance of Novel Composite and Integrated Devices Applied to Waste Heat Recovery," ASME J Heat Transfer, 135(3), 031706 (1-11). http://dx.doi.org/10.1115/1.4007892.

Rowe, D.M., editor, 2006, Thermoelectrics Handbook Macro to Nano, CRC Press, Taylor \& Francis Group, Boca Raton.

Smith, K., and Thornton, M., 2009, "Feasibility of Thermoelectrics for Waste Heat Recovery in Conventional Vehicles," Tech. rep., U.S. Department of Energy. www.nrel.gov/docs/fy09osti/44247.pdf.

Sootsman, J.R., Chung, D.K., and Kanatzidis, M.G., 2009, "New and Old Concepts in Thermoelectric Materials," Angew Chem Int Ed, 48, 86168639.

http://dx.doi.org/10.1002/anie.200900598.

Tritt, T.M., 2011, “Thermoelectric Phenomena, Materials, and Applications," The Annual Review of Materials Research, 41, 433-448. http://dx.doi.org/10.1146/annurev-matsci-062910-100453.

Yazawa, K., and Shakouri, A., 2012, "Cost-Effective Waste Heat Recovery Using Thermoelectric Systems," J Mater Res, 27(9), 1211-1217. http://dx.doi.org/10.1557/jmr.2012.79.

Ziolkowski, P., Poinas, P., Leszczynski, J., Karpinski, G., and Muller, E., 2010, "Estimation of Thermoelectric Generator Performance by Finite Element Modeling," Journal of Electronic Materails, 39(9), 1934-1943. http://dx.doi.org/10.1007/s11664-009-1048-0. 